\title{
Quantifying disorder in colloidal films spin-coated onto patterned substrates
}

\author{
Raheema Aslam, ${ }^{1}$ Sergio Ardanza-Trevijano, ${ }^{1,2}$ Kristin M. Poduska, ${ }^{3}$ Anand Yethiraj, ${ }^{3,{ }^{*}}$ and Wenceslao González-Viñas ${ }^{1,4, \dagger}$ \\ ${ }^{1}$ Universidad de Navarra, Complex Systems Group, Pamplona E-31008, Spain \\ ${ }^{2}$ Universidad de Navarra, Topology and FUZZY Logic Group, Pamplona E-31008, Spain \\ ${ }^{3}$ Department of Physics and Physical Oceanography, Memorial University of Newfoundland, St. John's, Newfoundland A1B 3X7, Canada \\ ${ }^{4}$ Universidad de Navarra, PHYSMED Group, Pamplona E-31008, Spain
}

(Received 11 December 2016; published 17 March 2017)

\begin{abstract}
Polycrystals of thin colloidal deposits, with thickness controlled by spin-coating speed, exhibit axial symmetry with local 4-fold and 6-fold symmetric structures, termed orientationally correlated polycrystals (OCPs). While spin-coating is a very facile technique for producing large-area colloidal deposits, the axial symmetry prevents us from achieving true long-range order. To obtain true long-range order, we break this axial symmetry by introducing a patterned surface topography and thus eliminate the OCP character. We then examine symmetryindependent methods to quantify order in these disordered colloidal deposits. We find that all the information in the bond-orientational order parameters is well captured by persistent homology analysis methods that only use the centers of the particles as input data. It is expected that these methods will prove useful in characterizing other disordered structures.
\end{abstract}

DOI: 10.1103/PhysRevE.95.032607

\section{INTRODUCTION}

Producing perfect, close-packed colloidal crystals is a cheap means to fabricate photonic band-gap materials [1-3]. Numerous techniques have been used to obtain well-ordered dried colloidal deposits [4-17]. However, it is challenging to obtain defect-free colloidal crystals over large areas. There is a growing realization [18] that nonequilibrium techniques might need to be employed to achieve better control of colloidal self-organization.

Spin-coating has recently been introduced in colloid science as a far-from-equilibrium modality to fabricate colloidal crystals [11,19-22]. It is very fast, reproducible, simple, and needs less material [20]. However, the axial symmetry of spin-coating makes the resultant colloidal films polycrystalline in a peculiar way, i.e., the orientationally correlated polycrystal (OCP) [11].

In spin-coating, the thickness and the uniformity of the films are important and strongly dependent on several control parameters, including the spin time and speed, the viscosity of fluids, the density and the evaporation rate of the fluids, the concentration of the suspension, as well as the substrate surface characteristics [20,23,24]. Electric fields have been applied during spin-coating of colloids to produce translationally ordered structures in Refs. [25,26]. Thus, if one can overcome the primary challenge set by the axial symmetry of spin coating, i.e., the emergence of OCPs, numerous ways to achieve better order would arise.

To achieve reliable and measurable improvements in order, it is crucial to characterize the degree of structural order and disorder systematically. Although there exist several methods for characterizing colloidal structures in real space [27-30], these translational- and orientational-order-based methods are more suited to homogeneous structures of reasonably high crystallinity. For example, a recent comparison of various

\footnotetext{
*ayethiraj@mun.ca

†wens@unav.es
}

different methods for making colloidal crystals [30,31] uses bond-orientational order parameters that can sensitively distinguish crystals for which $0.8<\Psi_{6}<1$.

In this manuscript, we address two goals. We first report a patterned surface topography technique to generate monolayer colloidal polycrystals, while at the same time eliminating the appearance of axial symmetry by spin-coating (i.e., the OCP character). The effect of surface patterning geometry on the morphology of spin-coated colloidal deposits is observed, and the influence of the scale spacing of the patterning topography on the structural order of colloidal deposits is presented.

Second, we introduce the use of persistent homology methods to examine structural heterogeneity in these colloidal polycrystals. One barrier to attempt at improving crystallinity by varying one of the many control parameters is the absence of reliable, quantitative measures of order in relatively poorly ordered colloidal films. Reciprocal-space methods, e.g., using small-angle and ultrasmall-angle scattering [32,33], are the standard for characterizing crystalline structures; however, spatial resolution is important both for identifying subtle differences in disordered structures, as well as for following the kinetics of crystallization. Real-space methods such as those discussed here can also be used in tandem with new developments in coherent $\mathrm{x}$-ray diffractive imaging [34]. In this work, we show that information about orientational order in spin-coated colloidal deposits, obtained by using orientationalorder based Minkowski structure metric [31,35,36], can be complemented by an examination of structural heterogeneity via persistent homology using the first Betti number [37].

\section{EXPERIMENTAL METHODS}

\section{A. Fabrication of patterned substrates}

We designed different photomask geometries to make triangular arrangements of equally sized regular hexagonal pillars, as shown in Fig. 1(a). The hexagon sides were $0.55 \mathrm{~mm}$. We call the distance between adjacent pillars the "scale spacing," and this was varied from 0.18 to $1.5 \mathrm{~mm}$, while 

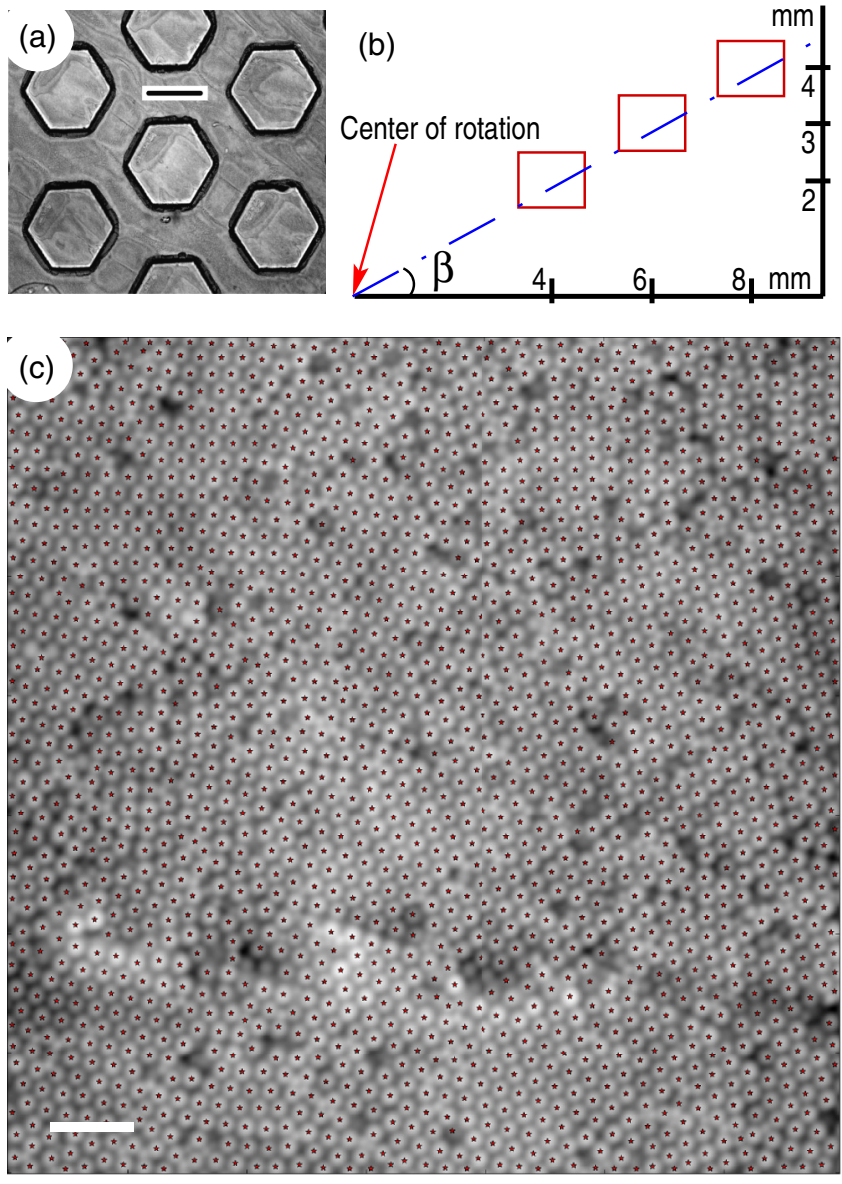

FIG. 1. (a) Optical microscope image of the top surface of a colloidal film deposited onto a patterned substrate (scale bar is $0.680 \mathrm{~mm}$ ). (b) Sketch of the AFM scanning regions (rectangles), at $2.24 \mathrm{~mm}$ intervals, where every scanned region makes an angle of $\beta=\arctan (1 / 2)$ with radial direction (dash-dotted lines) away from the center of rotation. (c) AFM image of a colloidal monolayer on the hexagonal pillar with the detected particle centers indicated as red marks (scale bar is $2 \mu \mathrm{m}$ ).

keeping the height of the pillars constant at $10 \mu \mathrm{m}$. To do so, SU-8, 2010 (UV-sensitive photoresist, MicroChem [38]) was spin-coated at $3000 \mathrm{rpm}$ for $30 \mathrm{~s}$ to achieve a thickness of $10 \mu \mathrm{m}$ on top of microscope cover slips $(22 \mathrm{~mm} \times$ $30 \mathrm{~mm}$ ). Thereafter, the templates were soft-baked at $95^{\circ} \mathrm{C}$ for 3 minutes and exposed to UV-light by using a maskless patterning system (Intelligent Micro Patterning LLC) for 3-4 minutes. Then, the templates were heated to $95^{\circ} \mathrm{C}$ for 3 minutes as a hard postexposure bake. The templates were then developed for 2 minutes with ethyl-lactate and a 5\% $\mathrm{NaOH}$ aqueous solution. This photolithography step enhanced the hydrophilic character of the template surfaces. Finally, the patterned cover-slip templates were glued onto commercially available microscope glass slides prior to performing the colloidal spin-coating experiments.

\section{B. Materials}

Silica particles of diameter $458 \pm 2 \mathrm{~nm}$ were dried overnight at $150^{\circ} \mathrm{C}$ in a convection oven to minimize the amount of absorbed moisture. After removal from the oven and cooling to room temperature, a volatile solvent (ethanol 95\%) was added to the particles to prepare a suspension with $20 \%(\mathrm{v} / \mathrm{v})$ concentration. Ultrasonication, typically for four hours, was then used to obtain a homogeneously dispersed suspension.

\section{Experiments}

Spin-coating experiments were performed in an air environment at room temperature with a commercial spin-coater (Laurell technologies, WS-650SZ-6NPP), which was housed in a fume hood to protect users from solvent vapors. When the patterned substrate reached a constant angular velocity (3000 rpm), $60 \mu \mathrm{L}$ of the colloidal suspension was pipetted onto it. The spinning was stopped when the dispersion had completely dried on the patterned substrate. A representative example of a dried monolayer colloidal film is shown in Fig. 1.

\section{Characterization}

The lithographic templates were inspected by optical microscopy (Nikon Eclipse 80-i upright microscope) prior to and after spin-coating. A representative optical micrography of a dried spin-coated film on a template is shown in Fig. 1(a). Atomic force microscopy (AFM, Asylum Research MFP-3D) was used to get images that we could use to get particle positions. Prior to AFM measurements, a thin layer $(\approx 100 \mathrm{~nm})$ of poly(methyl methacrylate) (PMMA) was spin-coated on top of the colloids to prevent the AFM tip from intermittently picking up — and then dropping - colloidal particles. The AFM was operated in contact mode. A representative AFM image in Fig. 1(c) shows the microscopic structure of the top surface of a monolayer colloidal deposit on top of an hexagonal pillar. We scanned and captured regions of $20 \times 20 \mu \mathrm{m}^{2}$ at regular intervals along the radial direction of each sample, choosing an angle that was not along any high-symmetry direction. We achieved this by making a series of $2 \mathrm{~mm}$ horizontal offsets, coupled with $1 \mathrm{~mm}$ vertical offsets, so that each successive image was equally along a radial direction that makes an angle of $\beta=\arctan (1 / 2) \approx 26.6^{\circ}$, away from the center of rotation. A schematic diagram is in Fig. 1(b).

\section{STRUCTURAL ANALYSIS METHODS}

To characterize colloidal structures in terms of their local order, we further quantify the AFM images of colloidal deposit on the hexagonal pillar in depth with different methods. To do so, in each AFM image, the positional coordinates of the particles are obtained [a sample of which is shown in Fig. 1(c) with tiny spots at the centroid of each detected particle], using home-made routines which are based on the scikit-image processing library [39]. First, we observed from the pair correlation function $g(r)$ that there is no long-range translational order in any of the deposits, and that they were qualitatively indistinguishable. Then, they were analyzed with bond-orientational order parameters (characterized by a Minkowski structure metric) and persistent homology techniques. Finally, we present a comparison of structural order in colloidal films based on these two kinds of analysis. 


\section{A. The Minkowski structure metric $\psi_{s}^{\mathrm{msm}}$}

The Minkowski structure metric (msm) is obtained by modifying the conventional local bond-orientational order parameters $\psi_{s}$ for a given weight $s$ [40]. $\psi_{s}$ calculated for particle $a$ was expected to serve well for a measure of $s$-fold symmetry in highly crystalline samples in the surrounding of the aforementioned particle. The most common arrangements in two-dimensional (2D) ordered structures are square $(s=4)$ and hexagonal $(s=6)$. As a matter of fact, some fundamental difficulties [36] were detected in this method. It assumes the geometrical arrangement of a set of nearest neighbors $\mathcal{N} N(a)$ around a particle (or vertex of the structure) $a$; where $\mathcal{N} N(a)$ has greater influence on $\psi_{s}(a)$ values specifically in the case of a square structure [41]. Also, the discrete nature of $\mathcal{N} N(a)$ is not a continuous function of the particle coordinates which is responsible for the lack of robustness of $\psi_{s}$ as structure metric. As a result, the Minkowski structure metric $(\mathrm{msm})$ $\psi_{s}^{m s m}$ is introduced to overcome these issues [31,35,36,42]. In the $m s m$, the contribution of each nearest neighbor to the structure metric is weighted by a relative length factor $l(\lambda) / L$, where $l(\lambda)$ is the length between two neighboring vertices of the Voronoi cell of particle $a$ that corresponds to a given bond $\lambda$ (or edge of the structure), and $L=\sum_{\lambda^{\prime} \in \mathcal{B}(a)} l\left(\lambda^{\prime}\right)$ is the total perimeter length of the Voronoi cell of particle $a$, as can be seen in Fig. 2. $\mathcal{B}(a)$ is the set of bonds which link particle $a$ with its nearest neighbors $\mathcal{N} N(a)$. This simple modification leads to a robust, continuous, and parameter-independent structure metric $\psi_{s}^{m s m}(a)$ which avoids the flaws of the bond order parameters $\psi_{s}$. The Minkowski structure metric $\psi_{s}^{m s m}(a)$ in $2 \mathrm{D}$ is defined as

$$
\psi_{s}^{m s m}(a)=\sum_{\lambda \in \mathcal{B}(a)} \frac{l(\lambda)}{\sum_{\lambda^{\prime} \in \mathcal{B}(a)} l\left(\lambda^{\prime}\right)} e^{i s \theta_{a b(\lambda)},}
$$

where $\theta_{a b(\lambda)}$ is the angle of the bond $\lambda$ (which links $a$ to $b$; see Fig. 2) with a reference axis.

\section{B. Persistent homology}

The $m s m$ successfully quantifies the degree of $s$-fold orientational order in crystalline structures. Nevertheless, the

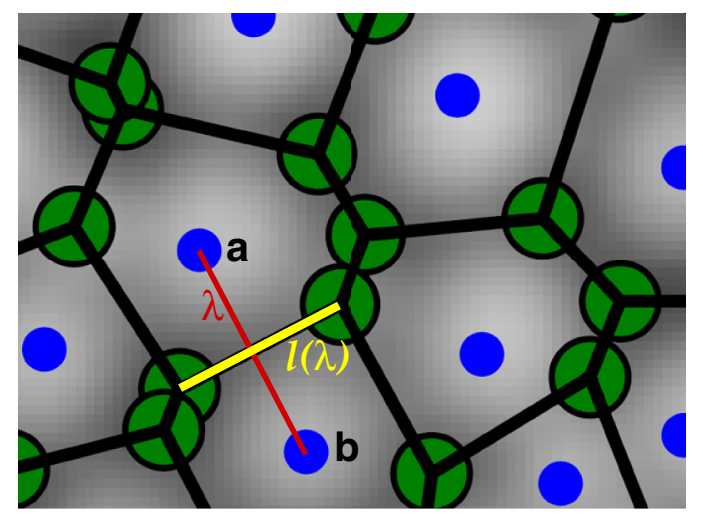

FIG. 2. Voronoi diagram, overlaid on part of an AFM image of a spin-coated deposit. The heavy yellow line $l(\lambda)$ shows the length between two neighboring vertices (large and open green circles) of the Voronoi cell of particle $a$ (small and solid blue dots) that corresponds to a given bond $\lambda$ with particle $b$. information given by it in the case of disordered systems is limited, and the order (or disorder) characterization has to be complemented by other, global, techniques.

Persistent homology is a tool with roots in algebraic topology that has been applied to extract topological information from geometric data. It has been notably successful in the analysis of particulate systems [37,43-48]. To describe what persistent homology measures, we first introduce the Vietoris-Rips complex. Then we give an intuitive idea of what the homology of a simplicial complex is and, finally, we describe persistent homology. For more details on homology and persistent homology we refer to Ref. [49].

Given a set of points (in our case the center of the particles), a metric (Euclidean distance), and a "filtration" parameter value $\delta$ we construct a two-dimensional simplicial complex, called the two-dimensional Vietoris-Rips complex (VRC) that has the set of points as vertices, an edge for each pair of vertices $v_{i}$ and $v_{j}$ such that $d\left(v_{i}, v_{j}\right)<\delta$, and a face (a triangle in this case) for each triplet of vertices with pairwise distance less than $\delta$. The resulting object with vertices, edges, and faces is the two-dimensional Vietoris complex that we denote $K(\delta)$. Note that, if $\delta_{1} \leqslant \delta_{2}, \mathrm{~K}\left(\delta_{1}\right) \subseteq K\left(\delta_{2}\right)$. An example of how the VRC changes for increasing values of the filtration parameter can be found in Fig. 3(a). Homology measures the number of "holes" in different dimensions in simplicial complexes. More concretely, given a simplicial complex $K$, homology assigns a sequence $H_{i}(K)$ of $\mathbb{Z}_{2}$-vector spaces, where $i$ is a non-negative integer. The dimension of the vector space $H_{0}(K)$ is called the zeroth Betti number $\beta_{0}$ and counts the number of connected components of our simplicial complex, and the dimension of

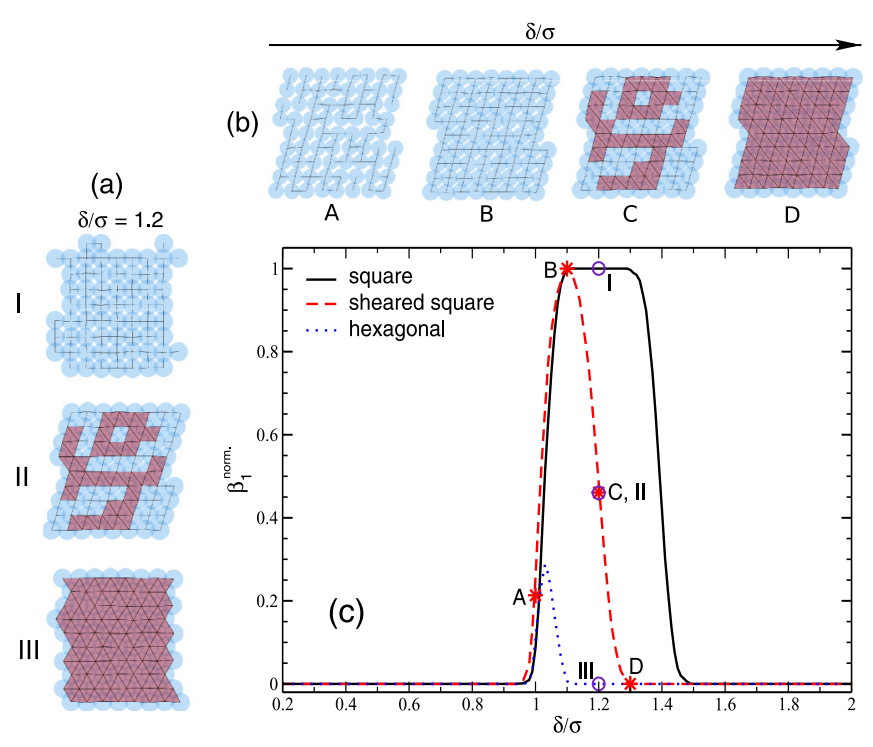

FIG. 3. Examples of persistence homology analysis on three compact artificial lattices (see text). (a) Vietoris-Rips complex (VRC) for square (I), sheared square [II, C in panel (b)] and hexagonal (III) at the same filtration parameter $(\delta=1.2 \sigma)$ [open circles in panel (c)]. (b) VRC for increasing values of filtration parameter $\delta$ [stars on dashed, red line in panel (c)], $\delta_{\mathrm{A}}=1 \sigma, \delta_{\mathrm{B}}=1.1 \sigma, \delta_{\mathrm{C}}=1.2 \sigma$, $\delta_{\mathrm{D}}=1.3 \sigma$. (c) $\beta_{1}^{\text {norm }}$ vs $\delta / \sigma$ for the three artificial lattices, where $\beta_{1}^{\text {norm }}$ is normalized in such a way that the maximum value for a perfect square lattice is 1 . The widest plateau appears for the square lattice. 
the vector space $H_{1}(K)$, which is called $\beta_{1}$, counts the number of loops in our graph that are not covered with triangles (the number of holes or uncovered loops in the two-dimensional complex). We can find bases of these vector spaces which correspond to the loops that we are counting.

Now we are ready to give an idea of what persistent homology is. For $\delta_{1}<\delta_{2}$, the inclusion $K\left(\delta_{1}\right) \subseteq K\left(\delta_{2}\right)$ induces a linear map in each dimension $H_{i}\left(K\left(\delta_{1}\right)\right) \mapsto H_{i}\left(K\left(\delta_{2}\right)\right)$. Given a maximum value of the filtration parameter $\delta_{\max }$, the collection of all the simplicial complexes $K(\delta), 0 \leqslant \delta \leqslant \delta_{\max }$ is called a filtered complex. The persistent homology will capture the information of the homology groups (in our case $\mathbb{Z}_{2}$-vector spaces) for all possible values of the filtration parameter between 0 and $\delta_{\max }$ giving us a persistence diagram with the birth and death of each connected component in dimension 0 or the birth and death of each loop in dimension 1 . Notice that a persistence diagram is just a collection of intervals in each dimension, and hence it is sometimes referred to as a "bar code." For simplicity, we proceed to explain the $\beta_{0}$ case. We order all the vertices. Each represents a connected component at $\delta=0$ and hence $\beta_{0}(0)$ is the number of vertices. As the filtration parameter $\delta$ is increased, each component will "die" at the $\delta$ value where it gets connected with a point that appears earlier in the order. Hence, at $\delta=0, K(0)$ will have as many connected components as the number of points. The number of connected components will decrease as $\delta$ increases until everything is connected (provided $\delta_{\max }$ is large enough) and we have only one component, i.e., $\beta_{0}=1$.

We will not work with persistent diagrams, but with a summary called the Betti profile, a graph that represents, for each dimension, the corresponding Betti number as a function of $\delta$. In particular the one-dimensional Betti profile presents the number of loops $\left[\beta_{1}(\delta)\right]$ that we have as $\delta$ varies from 0 to $\delta_{\max }$. A series of free packages are available for computing persistent homology [50-52]. Here, we use the R package TDA using "DIONYSUS" to perform persistent homology calculations [53].

In Fig. 3 we provide a simple example of the above explanation for persistent homology measurements, using data given by the centers of particles extracted from three different artificially generated 2D lattices with low noise. We compare compact square (I), sheared square (II), and hexagonal (III) lattices, with a $10 \%$ additive noise to each coordinate of the particle centers and with a nearest-neighbor separation of $\sigma=10$ pixels. VRC diagrams [Fig. 3(a)] at the same filtration parameter $\delta$ distinguish among these three different structural orders. The effect of increasing the filtration parameter values above the diameter of the particles has two possible results, as shown in Fig. 3(b), using the example of a sheared square artificial lattice. At moderately high filtration-parameter values, new connections may lead to the creation of a larger number of loops (one-dimensional holes), as shown in part B; this corresponds to higher value of $\beta_{1}$ in Fig. 3(c). At even higher filtration parameter values, the lattice is covered by triangles, as shown in parts $\mathrm{C}$ and $\mathrm{D}$; this corresponds to a decrease in $\beta_{1}$ in Fig. 3(c).

Profiles of the first Betti number $\beta_{1}$ for artificial lattices [square, sheared square, and hexagonal; Fig. 3(c)] show that the lowest $\beta_{1}$ peak height corresponds to the hexagonal structure, and the widest plateau is associated with the square structure. For all lattices, the rise in $\beta_{1}$ corresponds to a filtration parameter $\delta$ that equals the nearest-neighbor distance. For an almost perfect square lattice, the $\beta_{1}$ peak exhibits a plateau that decreases abruptly beyond the next-nearest distance (at $\delta / \sigma \approx \sqrt{2}$ ). For sheared square lattices, which are intermediate between square and hexagonal $(50 \%)$, the sudden decrease of $\beta_{1}$ occurs for $\delta / \sigma \approx(1+\sqrt{3} / 4)^{1 / 2}$. Furthermore, its plateau collapses into a peak that is narrower. For sheared structures that are closer to hexagonal, the $\beta_{1}$ peak value decreases below 1 .

In summary, we use persistent homology analyses to extract three quantities that describe the structure of colloidal films (see Sec. IV, especially Figs. 7 and 8 and text): the value of $\delta / \sigma$ at which $\beta_{1}$ first becomes nonzero, the height of the $\beta_{1}$ peak, and the width of the $\beta_{1}$ peak plateau (or peak).

\section{RESULTS AND DISCUSSIONS}

\section{A. Qualitative aspects}

A series of experiments were performed in order to examine the role played by a patterned substrate on the spin-coated deposit. An unpatterned substrate, acted as the control sample; it was coated with SU-8 to have comparable wettability to the patterned substrates. Figure 4(a) shows a colloidal sediment on an unpatterned substrate, while Figs. 4(b)-4(d) show sediments on substrates that were patterned with a lattice of hexagonal pillars with scale spacing of $0.3,0.55$, and $0.82 \mathrm{~mm}$, respectively. The unpatterned substrates [Fig. 4(a)] produce deposits with large-scale orientationally correlated polycrystalline (OCP) character that exhibits colored arms [11], as well as large cracks or big holes. In contrast, the patterned substrates produce polycrystalline deposits that do
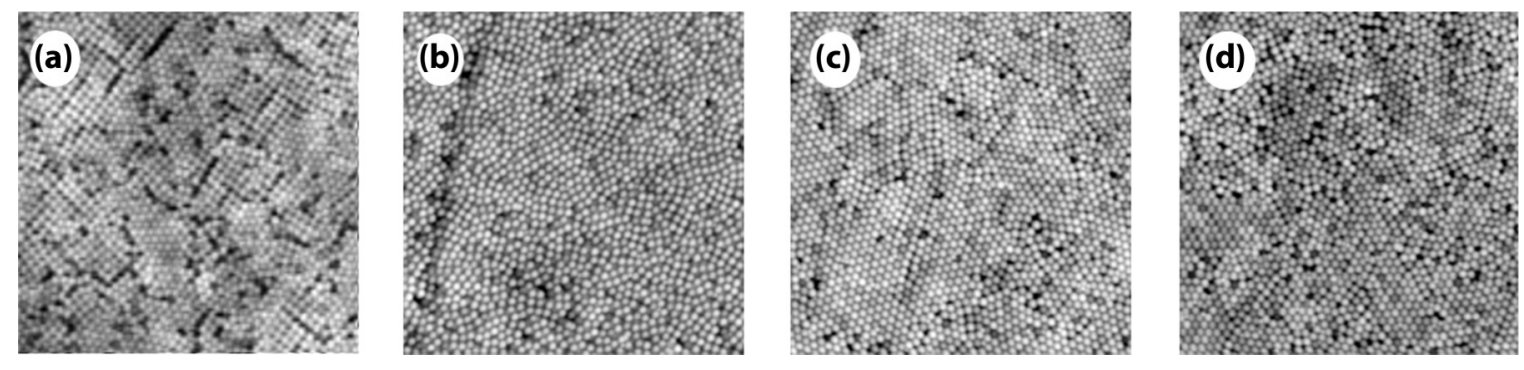

FIG. 4. AFM images of colloidal deposits, obtained at the same distance [(4,2) mm] from the spinning center of each sample. (a) An unpatterned substrate produces deposits with OCP behavior. (b)-(d) Patterned substrates remove any OCP behavior, at intermediate scale spacing between hexagonal pillars: (b) $0.3 \mathrm{~mm}$, (c) $0.55 \mathrm{~mm}$, and (d) $0.82 \mathrm{~mm}$. All images are $20 \mu \mathrm{m} \times 20 \mu \mathrm{m}$. 
not show evidence of the orientation correlation that is typical for spin-coated colloidal crystals [Figs. 4(b)-4(d)].

Preliminary experiments showed that the OCP character disappeared only for intermediate scale spacing $(0.30$ $0.82 \mathrm{~mm}$ ). At higher and lower scale spacing, the surface appears to function similar to an unpatterned substrate for the drying suspension. This dependence of the structural order on the scale spacing may be related to hydrodynamics or surface tension. Intermediate-spaced hexagonal pillars behave as obstacles that prevent the fluid flows that are responsible for OCP behavior. We note that all subsequent results discussed here are restricted to the range of scale spacings where the OCP does not appear. These data are also compared with results from unpatterned substrates.

In what follows, the positions of colloidal particles, extracted from AFM images, are analyzed in detail to assess 4-fold and 6-fold structural ordered regions separately using different methods: Minkowski structure metric analysis and persistent homology.

\section{B. Minkowski structure metric analysis}

The colloid positions derived from AFM images allow us to obtain the (complex) Minkowski structure metric $\psi_{s}^{m s m}$ for both 4-fold and 6-fold symmetry ( $s=4$ or 6 ), and for each particle independently. Then, its absolute value $\left|\psi_{s}^{m s m}\right|$ and $\operatorname{argument} \arg \left(\psi_{s}^{m s m}\right)$ provide useful information about the local $s$-fold degree of order and local orientation, respectively.

Figure 5 shows a plot of $\left\langle\left|\psi_{4}^{m s m}\right|\right\rangle$ vs $\left\langle\left|\psi_{6}^{m s m}\right|\right\rangle$ for regions at different distances from the spinning center, where the average is over all particles in a given image. For example, the AFM

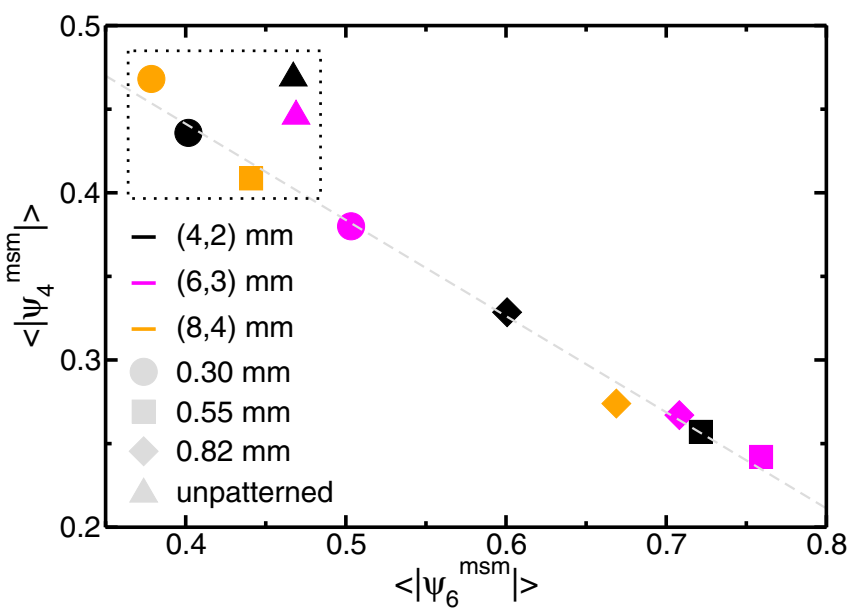

FIG. 5. Average Minkowski structure metric values $\left\langle\left|\psi_{6}^{m s m}\right|\right\rangle$ and $\left\langle\left|\psi_{4}^{m s m}\right|\right\rangle$ for particle positions extracted from AFM images of spincoated deposits. The three different scale spacings, $0.3,0.55$, and 0.82 $\mathrm{mm}$, are represented by circles, squares, and diamonds, respectively, while AFM images at distances corresponding to vectors $(4,2) \mathrm{mm}$, $(6,3) \mathrm{mm}$, and $(8,4) \mathrm{mm}$ (all at the same polar angle but at the distances $4.47,6.71$, and $8.83 \mathrm{~mm}$ from the center) are represented by black, dark gray (magenta), and light gray (orange) symbols, respectively. For the scale spacing of $0.55-0.82 \mathrm{~mm}$ we have greater values for $\left\langle\left|\psi_{6}^{m s m}\right|\right\rangle$ and smaller for $\left\langle\left|\psi_{4}^{m s m}\right|\right\rangle$. The dashed line shows a linear fit for data. The results for unpatterned substrates are shown as well (triangle symbols) but are not considered in the fit. images shown in Fig. 4, at a distance $(4,2) \mathrm{mm}$ from the center, are represented in this plot by black triangle [Fig. 4(a)], circle [Fig. 4(b)], square [Fig. 4(c)], and diamond [Fig. 4(d)].

There is no clear systematic dependence of $\left\langle\left|\psi_{s}^{m s m}\right|\right\rangle$ on the distance from the center of spinning: the intermediate distance $(6,3) \mathrm{mm}$ appears to yield the highest value of $\left\langle\left|\psi_{6}^{m s m}\right|\right\rangle$. In addition, there is no optimal scale spacing, since an intermediate value $(0.55 \mathrm{~mm}$, squares $)$ appears to yield the best order at two radial distances [dark gray (magenta) and black squares], but not for the third [light gray (orange) square].

For all samples that were spin-coated onto patterned substrates, there is, however, a strong correlation between $\left\langle\left|\psi_{6}^{m s m}\right|\right\rangle$ and $\left\langle\left|\psi_{4}^{m s m}\right|\right\rangle$ which is shown by the dashed line in Fig. 5 (linear fit with absolute value of correlation coefficient 0.995 ). We do not understand the origin of the linear relation between $\left\langle\left|\psi_{6}^{m s m}\right|\right\rangle$ and $\left\langle\left|\psi_{4}^{m s m}\right|\right\rangle$ in this specific kind of experiment, but it is worth noting that the relationship is statistically significant. This correlation implies a trend that needs to be explored further.

Although the points from the unpatterned substrate (triangles) are not in the confidence interval of the linear model (confidence $>0.999$ ), they lie very close to those of the patterned substrates with highest $\left\langle\left|\psi_{4}^{m s m}\right|\right\rangle$ and lowest $\left\langle\left|\psi_{6}^{m s m}\right|\right\rangle$ (dashed rectangle in Fig. 5). At this point, it is not clear if we can robustly distinguish the differences in disorder [refer to Figs. 4(a) and 4(b)] by using the msm method, despite the fact that the structures are very different; namely, OCP $v$ s not OCP. Thus, it is more useful to classify these structures through the distribution of the cells' orientation with respect to the radial direction, rather than by means of the orientational degree of order.

Hence, we show histograms of the phase of $\psi_{6}^{m s m}$ and $\psi_{4}^{m s m}$ for AFM images of samples from experiments using unpatterned substrates which lead to OCP behavior [Figs. 6(a)
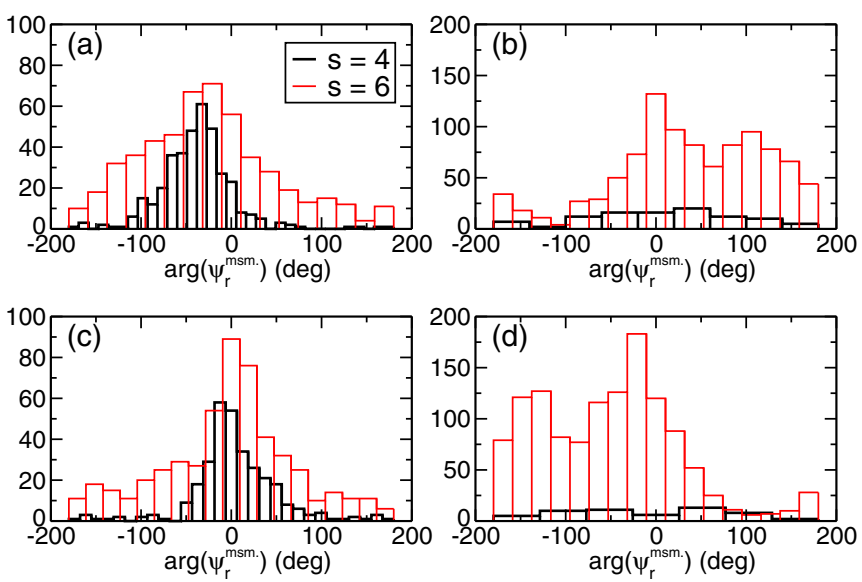

FIG. 6. Histograms of the local phases of $\psi_{6}^{m s m}$ and $\psi_{4}^{m s m}$ that are obtained in AFM images taken at different distances from the rotation center [(4,2) mm for panels (a) and (b); and (6,3) mm for panels (c) and (d)]. Gray thin (red) bins correspond to 6-fold symmetries and black thick bins to 4-fold symmetries. (a), (c) Using unpatterned substrates where crystalline domains are aligned along the radial direction, i.e., with OCP character. Here the histograms are narrower. (b), (d) Using a patterned substrate with $0.82 \mathrm{~mm}$ spacing between hexagon pillars without OCP character. Here the histograms are broader. 
and 6(c)], and patterned substrates which do not lead to OCP character [Figs. 6(b) and 6(d)]. In the histograms, we only select the particles which have $\left|\psi_{6}^{m s m}\right| \geqslant 0.5$ and $\left|\psi_{4}^{m s m}\right| \geqslant 0.6$ for 6- and 4-fold symmetry, respectively.

Here, gray thin (red) bins correspond to $\psi_{6}^{m s m}$ symmetries and black thick bins to $\psi_{4}^{m s m}$ symmetries. In Figs. 6(b) and 6(d), the images from top to bottom indicate histograms at increasing distances along the radial direction for the same experiment (patterned substrate with $0.82 \mathrm{~mm}$ spacing between pillars). Here, one sees a broad distribution of angles for both $\psi_{4}^{m s m}$ and $\psi_{6}^{m s m}$, which impede the OCP character. However, for the unpatterned substrate [Figs. 6(a) and 6(c)] both the $\psi_{4}^{m s m}$ and $\psi_{6}^{m s m}$ evince sharper angle distributions around the radial direction $\left(0^{\circ}\right)$. It is, thus, clear that one can have long-range OCP structure even when the local bond order parameters are small, e.g., $\psi_{4}^{m s m}<0.6$ and $\psi_{6}^{m s m}<0.5$ for the unpatterned substrates (Fig. 5, triangles).

\section{Persistent homology analysis}

Since the local order in 2D systems is almost 6-fold or 4-fold, local measures like the Minkowski (bond-orientational order parameter) analysis may not provide enough information to distinguish different kinds of structural disorder [54]. The goal here is to test persistent homology techniques by using parametrized first Betti number with filtration parameter $\delta$ to analyze the disorder in structure of our 2D spin-coated colloidal films in view of what we learned from a look at wellordered artificial lattices (Fig. 3 and corresponding text). First, we show the Vietoris-Rips complex for increasing values of $\delta$ [Fig. 7(a)], for structures on unpatterned (left) and patterned (right) substrates. Hexagonal domains are seen as regions with cells covered by triangles [light gray (pink)]. It is seen that the number of loops (empty polygons with more than three sides) decreases significantly in all examples as one goes from a filtration parameter $\delta$ of $1.2 \sigma$ to $1.8 \sigma$. The corresponding pair correlation function in Fig. 7(b) shows that this corresponds to a distance that is well beyond the first peak of the $g(r)$.

In Fig. 8(a) we represent the number of loops, $\beta_{1}^{\text {norm }}$, for increasing filtration parameter $\delta$ for several experiments [data at $(4,2) \mathrm{mm}$ from the center of rotation]. $\beta_{1}^{\text {norm }}$ is normalized in such a way that the maximum value for a perfect square lattice is 1 . The four $\beta_{1}^{\text {norm }}$ curves are obtained from VRCs such as the one in Fig. 7(a).

The width of each $\beta_{1}^{\text {norm }}$ vs $\delta / \sigma$ curve at $10 \%$ below its maximum value is another useful measure of the heterogeneous openness of the structure, and it is represented in Fig. 8(a) as well. We have seen, in our example with artificial lattices [Fig. 3(c)] that the lowest $\beta_{1}^{\text {norm }}$ peak corresponds to hexagonal structure. In the example in Fig. 8(a), we see, consistently with the artificial lattices, that all the $\beta_{1}$ profiles for all the patterned samples have the same rising slopes, and the sample with the highest $\left\langle\left|\psi_{6}^{m s m}\right|\right\rangle$ shown in Fig. 5 has the lowest $\beta_{1}^{\text {norm }}$ peak. Similarly, as we saw for artificial lattices [Fig. 3(c)], the widest plateau corresponds to square structure. Therefore, the structure atop the unpatterned substrate [black dotted curve of Fig. 8(a)] shows the strongest 4 -fold order. In that case, we observe that the rising slope is smaller relative to the case of patterned substrates, where the presence of hexagonal pillars forces more compact structures. (a)
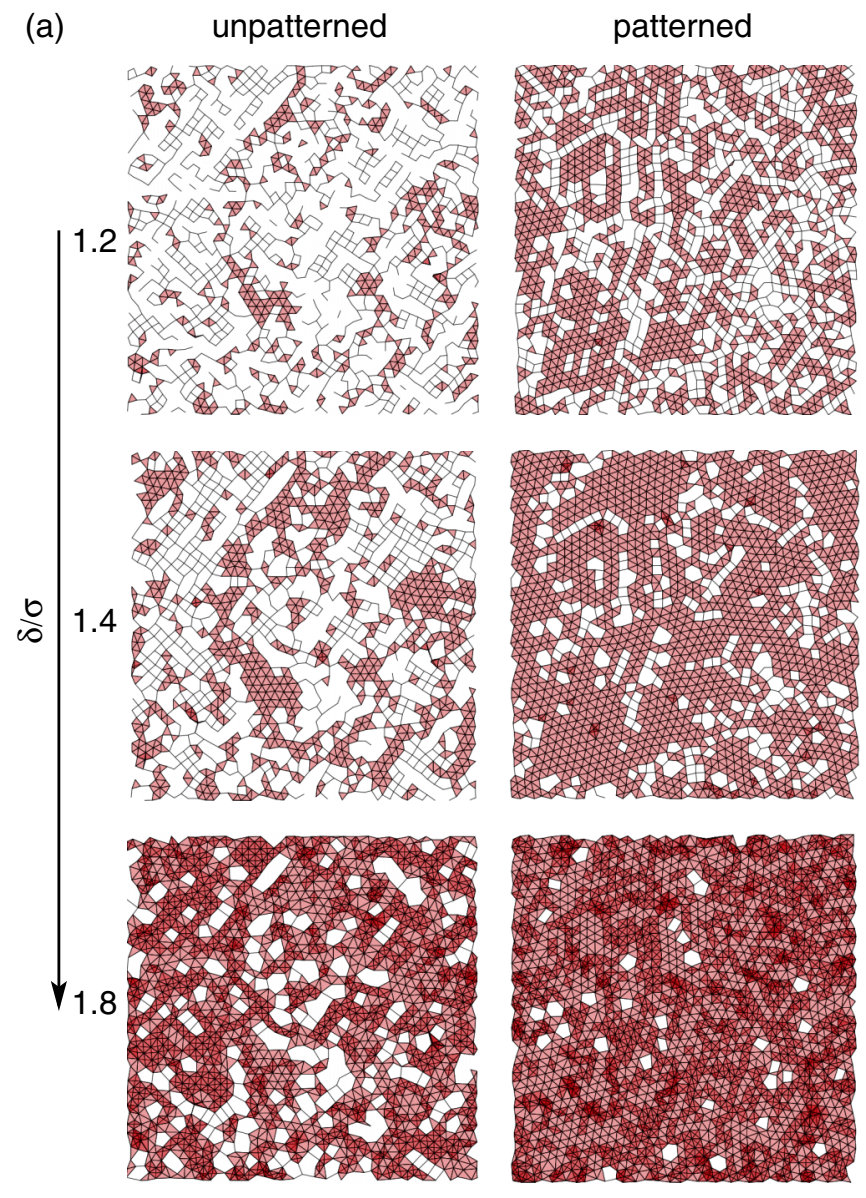

(b)

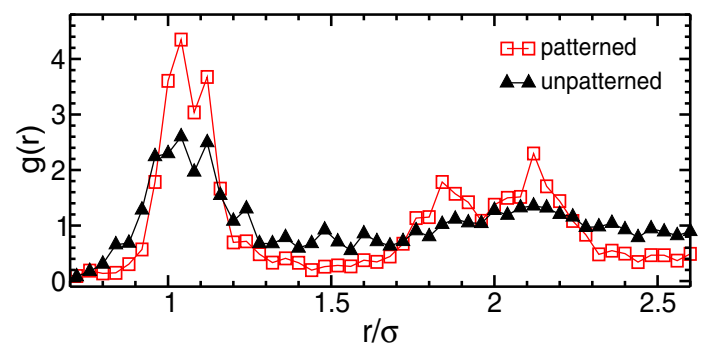

FIG. 7. (a) Vietoris-Rips complex for three values of the filtration parameter for a unpatterned substrate (left) and for a scale spacing of $0.55 \mathrm{~mm}$ (right) both at the radial distance $(4,2) \mathrm{mm}$ from the center of rotation. $\delta$ is the filtration parameter and $\sigma$ is the particle diameter. (b) In disordered lattices, a good quantifier for nearest-neighbor distance is the position of the first peak of the pair correlation function $g(r)$ (seen to be at about $1.05 \sigma$ ). Thus, $r=1.2 \sigma, 1.4 \sigma$, and $1.8 \sigma$ exceed the nearest-neighbor distance and are thus reasonable values to use for the filtration parameter.

In Fig. 8(b), we plot the maximum of the $\beta_{1}$ curve against its width. At the top right, the black circle (scale spacing of $0.3 \mathrm{~mm}$ ) corresponds to the Vietoris-Rips complex (VRC) in Fig. 8(c): here, good hexagonal order (pink shaded regions) barely exists. At the bottom right of Fig. 8(b), the black triangle (unpatterned substrate) corresponds to the VRC in Fig. 8(d). We see that both the white and pink shaded domains are spatially correlated in Fig. 8(d) and less so in Fig. 8(c). Moreover, in the latter there are other shapes (=disorder), but their areas are similar to the squares. In the former, we have 


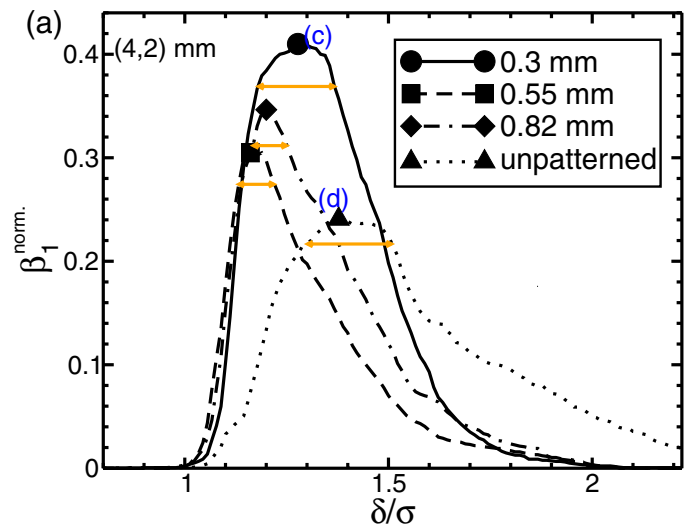

(c)
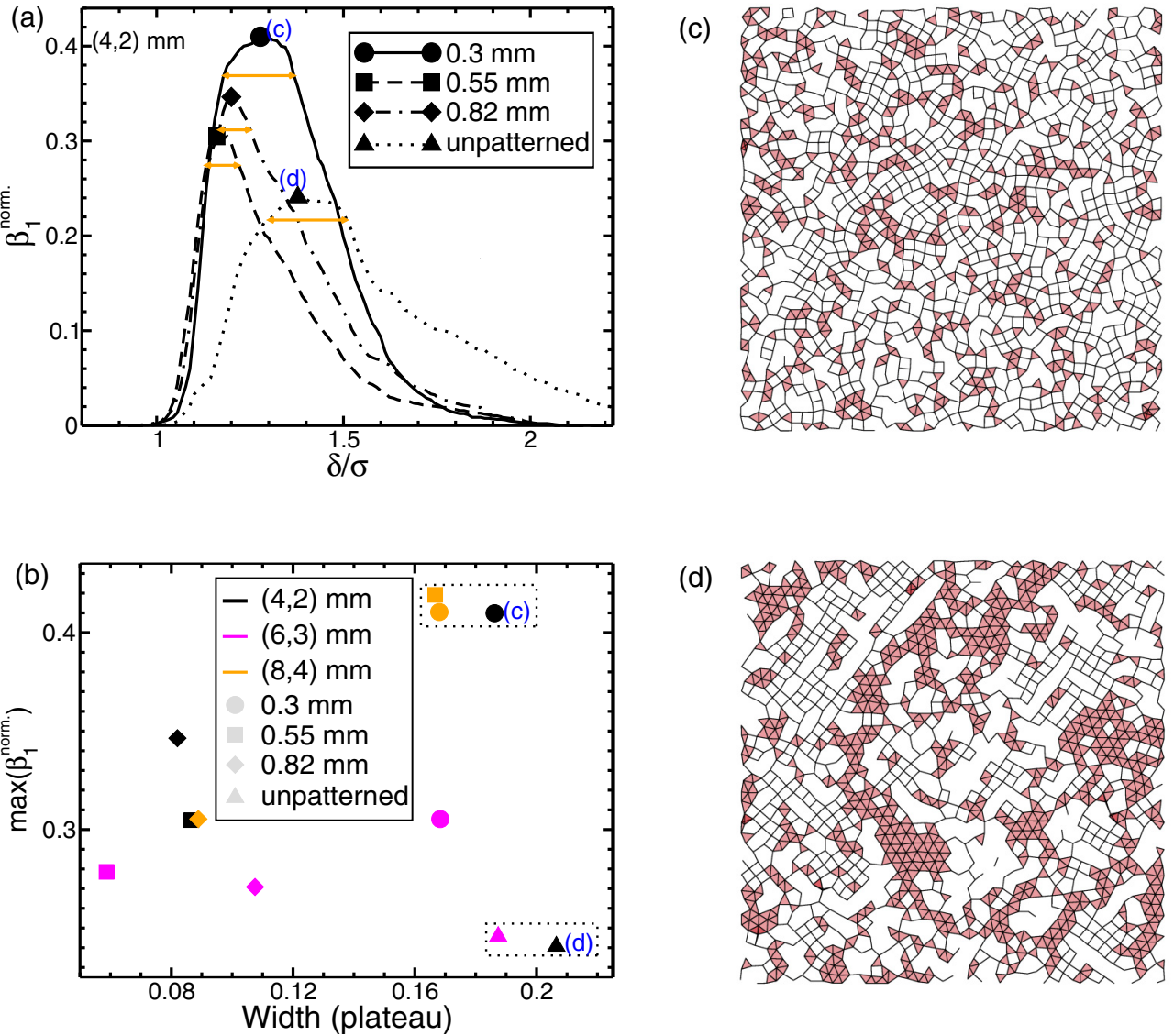

(d)

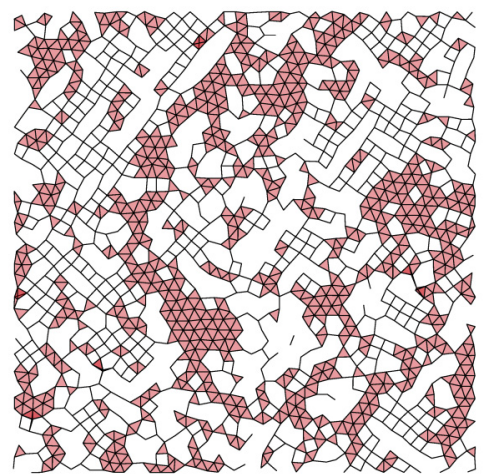

FIG. 8. Persistent homology analysis of the experimental results. (a) As in Fig. 3, $\beta_{1}^{\text {norm }}$, which represents the normalized number of loops (one-dimensional holes), versus the normalized filtration parameter $\delta / \sigma . \sigma$ is the mean size of the particles in the AFM images. For clarity, only the curves for $(4,2) \mathrm{mm}$ results are included. Recall that a wider plateau means more square structure and a sharper peak means more hexagonal structure. (b) Summary of persistent homology analysis by using the maximum of $\beta_{1}^{\text {norm }}$ curves as a function of their width (measured at $10 \%$ below the peak value of the curve). All data points are included. Symbols and colors correspond to those in Fig. 5. (c) Vietoris-Rips complex (VRC) for the experiment $0.3 \mathrm{~mm}$ of scale spacing at $(4,2) \mathrm{mm}$ from the center of rotation. The filtration parameter corresponds to the maximum value of the corresponding $\beta_{1}^{\text {norm }}$ curve. It is indicated as (c) in panels (a) and (b). The field of view is $20 \mu \mathrm{m}$. (d) Vietoris-Rips complex (VRC) for the unpatterned experiment at $(4,2) \mathrm{mm}$ from the center of rotation. The filtration parameter corresponds to the maximum value of the corresponding $\beta_{1}^{\text {norm }}$ curve. It is indicated as (d) in panels (a) and (b). The field of view is also $20 \mu \mathrm{m}$.

an appreciable amount of bigger holes which are related to cracks or local lack of compactness. All these features are quantitatively shown in Fig. 8(b).

\section{A COMPARISON OF ANALYSIS TECHNIQUES}

Minkowski analysis that involves local measures, such as Voronoi cells, defines local order in 2D systems as 6-fold or 4-fold. However, we show in this work that the Minkowski analysis is not sensitive enough to structural disorder in colloidal crystals that we induced by changing substrate wettability and scale spacing.

The persistent homology analysis method is introduced here in the analysis of colloidal order and disorder. A perfect triangular lattice will produce the smallest $\beta_{1}$, and the perfect square lattice will produce the largest $\beta_{1}$, which will last from the value of the filtration parameter $\delta$ at which the squares are first detected (length of the side) until the value $\delta$ that is equal to length of the diagonal of the square. For a slightly sheared square lattice instead of a perfect square lattice, $\beta_{1}$ achieves its maximum value for a shorter range of filtration parameters. This trend stops when the filtration reaches the length of the shortest diagonal of the diamonds. This means that structures with the same $\beta_{1}$ magnitude for low filtration values (which are comparable to the size of the particles themselves) can be distinguished with an appropriate higher value of the filtration parameter. Thus, square domains can be distinguished by applying different filtration parameter values.

The key advantage of the persistent homology method in this study is highlighted in a comparison of Figs. 5 and 8(b). Points that are very close in bond-order correlations, and highlighted in Fig. 5 with a dotted rectangle are appreciable separated in Fig. 8(b) [the collection of proximate points that are enclosed by the dotted rectangle in Fig. 5 are in fact in the top and bottom right in Fig. 8(b)].

\section{CONCLUDING REMARKS}

This work is admittedly not about the high degree of order in colloids spin-coated onto patterned substrates: we found that 
the patterns, at least with the geometry and pattern spacing considered in this work, yielded colloidal films with high degrees of disorder. Instead, this work deals with strategies to assess the disorder, as a precursor to improving specific structures in future work. In this study, two methods to characterize disorder in colloids structure spin-coated on patterned and unpatterned substrates-Minkowski measures of bond-orientational order and persistent homology-are compared. These analysis methods present a complementary and unified strategy for comparing degree of structural disorder. We find that structures very close in the $\left\langle\left|\psi_{6}^{m s m}\right|\right\rangle-\left\langle\left|\psi_{4}^{m s m}\right|\right\rangle$ space (Fig. 5, dotted-line box) can be properly distinguished in the $\operatorname{width}\left(\beta_{1}^{\text {norm }}\right)-\max \left(\beta_{1}^{\text {norm }}\right)$ space [Fig. 8(b), dottedline boxes], which also provides an intuitive explanation of the differences in the observed disorder [Figs. 8(c) and $8(\mathrm{~d})]$.

Spin-coating of concentrated colloids on patterned substrates breaks up the axial symmetry (eliminates the emergence of color arms). Polycrystals of monolayer on hexagon pillars are obtained. We found that scale spacing between hexagons is an important parameter to control the process of structure formation. For a range of substrate pattern spacing, we find that we can clearly free the system of the long-range orientational (OCP) structure that is characteristic of colloid spin-coating.

Minkowski analysis that involves local measures such as Voronoi cells defines local order in 2D systems as 6-fold or 4-fold and is less sensitive to structural disorder which in this work is influenced by substrate wettability and changes in the scale spacing.

This work has shown that persistent homology techniques can be used for distinguishing disorder in colloidal systems of spherical particles. We suggest that these methods can be extended to identify the structures of systems of nonspherical colloidal particles in liquid as well in solid phases, or even in glasses.

\section{ACKNOWLEDGMENTS}

R.A. acknowledges training assistance in use of the Atomic Force Microscopy from Payam Bagheri and financial support from the "Asociación de Amigos de la Universidad de Navarra." This work is partly supported by the Spanish Ministerio de Economía y Competitividad (Grants No. FIS201454101-P and No. MTM2016-79422-P), by FEDER funds, and by the Natural Sciences and Engineering Research Council of Canada.
[1] D. Norris and Y. Vlasov, Adv. Mater. 13, 371 (2001).

[2] P. Braun and P. Wiltzius, Nature (London) 402, 603 (1999).

[3] J. Joannopoulos, Nature (London) 414, 257 (2001).

[4] A. S. Dimitrov and K. Nagayama, Langmuir 12, 1303 (1996).

[5] S. H. Park, D. Qin, and Y. Xia, Adv. Mater. 10, 1028 (1998).

[6] P. Jiang, J. F. Bertone, K. S. Hwang, and V. L. Colvin, Chem. Mater. 11, 2132 (1999).

[7] C. Brinker, Y. Lu, A. Sellinger, and H. Fan, Adv. Mater. 11, 579 (1999).

[8] Z. Z. Gu, A. Fujishima, and O. Sato, Chem. Mater. 14, 760 (2002).

[9] Q. Li, Y. Chen, and P. Dong, Mater. Lett. 59, 3521 (2005).

[10] F. Meseguer, Colloids Surf., A 270-271, 1 (2005).

[11] C. Arcos, K. Kumar, W. González-Viñas, R. Sirera, K. M. Poduska, and A. Yethiraj, Phys. Rev. E 77, 050402(R) (2008).

[12] D. Nagao, R. Kameyama, H. Matsumoto, Y. Kobayashi, and M. Konno, Colloids Surf., A 317, 722 (2008).

[13] Y. Fu, Z. Jin, Z. Liu, Y. Liu, and W. Li, Mater. Lett. 62, 4286 (2008).

[14] A. J. Wang, S. L. Chen, P. Dong, C. T. Hu, and L. Sang, Thin Solid Films 519, 1798 (2011).

[15] V. E. Ferry, M. A. Verschuuren, M. C. van Lare, R. E. I. Schropp, H. A. Atwater, and A. Polman, Nano Lett. 11, 4239 (2011).

[16] C. Zhou, J. Han, and R. Guo, J. Colloid Interface Sci. 397, 80 (2013).

[17] A. Coll, S. Bermejo, D. Hernández, and L. Castañer, Nanoscale Res. Lett. 8, 26 (2013).

[18] J. Dobnikar, A. Snezhko, and A. Yethiraj, Soft Matter 9, 3693 (2013).

[19] P. Jiang and M. McFarland, J. Am. Chem. Soc. 126, 13778 (2004).
[20] Y. L. Wu, Ph.D. thesis, Utrecht University, Utrecht, The Netherlands, 2007, http://web.science.uu.nl/scm/Theses/ Wu_Thesis_2007color.pdf.

[21] L. T. Shereda, R. G. Larson, and M. J. Solomon, Phys. Rev. Lett. 101, 038301 (2008).

[22] A. Mihi, M. Ocaña, and H. Míguez, Adv. Mater. 18, 2244 (2006).

[23] M. Giuliani, Ph.D. thesis, University of Navarra, Pamplona, Spain, 2010, http://hdl.handle.net/10171/13612.

[24] M. Pichumani, Ph.D. thesis, University of Navarra, Pamplona, Spain, 2012, http://hdl.handle.net/10171/27662.

[25] J. Juárez and M. Bevan, J. Chem. Phys. 131, 134704 (2009).

[26] A. P. Bartlett, M. Pichumani, M. Giuliani, W. González-Viñas, and A. Yethiraj, Langmuir 28, 3067 (2012).

[27] S. Portal-Marco, M. À. Vallvé, O. Arteaga, J. Ignés-Mullol, C. Corbella, and E. Bertran, Colloids Surf., A 401, 38 (2012).

[28] M. J. McDonald, A. Yethiraj, and L. Y. Beaulieu, Meas. Sci. Technol. 23, 045606 (2012).

[29] A. J. Krejci, C. G. W. Thomas, and J. H. Dickerson, Phys. Rev. E 87, 042307 (2013).

[30] P. Bagheri, A. M. Almudallal, A. Yethiraj, and K. M. Poduska, Langmuir 31, 8251 (2015).

[31] P. Bagheri, A. M. Almudallal, A. Yethiraj, and K. M. Poduska, Langmuir 32, 2524 (2016).

[32] J. H. J. Thijssen, A. V. Petukhov, D. C. 't Hart, A. Imhof, C. H. M. van der Werf, R. E. I. Schropp, and A. van Blaaderen, Adv. Mater. 18, 1662 (2006).

[33] P. Huber, O. Bunk, U. Pietsch, M. Textor, and T. Geue, J. Phys. Chem. B 114, 12473 (2010).

[34] A. G. Shabalin, J.-M. Meijer, R. Dronyak, O. M. Yefanov, A. Singer, R. P. Kurta, U. Lorenz, O. Y. Gorobtsov, D. Dzhigaev, S. Kalbfleisch, J. Gulden, A. V. Zozulya, M. Sprung, A. V. Petukhov, and I. A. Vartanyants, Phys. Rev. Lett. 117, 138002 (2016). 
[35] A. Yethiraj, A. Wouterse, B. Groh, and A. van Blaaderen, Phys. Rev. Lett. 92, 058301 (2004).

[36] W. Mickel, S. C. Kapfer, G. E. Schröder-Turk, and K. Mecke, J. Chem. Phys. 138, 044501 (2013).

[37] S. Ardanza-Trevijano, I. Zuriguel, R. Arévalo, and D. Maza, Phys. Rev. E 89, 052212 (2014).

[38] MicroChem, NANO ${ }^{\mathrm{TM}}$ SU-8, Negative Tone Photoresist Formulations 50-100, Datasheet (2002).

[39] S. van der Walt, J. L. Schönberger, J. Nunez-Iglesias, F. Boulogne, J. D. Warner, N. Yager, E. Gouillart, T. Yu, and the scikit-image contributors, PeerJ 2, e453 (2014).

[40] P. J. Steinhardt, D. R. Nelson, and M. Ronchetti, Phys. Rev. B 28, 784 (1983).

[41] S. Casado, W. González-Viñas, and H. Mancini, Phys. Rev. E 74, 047101 (2006).

[42] S. C. Kapfer, W. Mickel, K. Mecke, and G. E. Schröder-Turk, Phys. Rev. E 85, 030301 (2012).

[43] M. Kramár, A. Goullet, L. Kondic, and K. Mischaikow, Phys. Rev. E 87, 042207 (2013).

[44] M. Kramár, A. Goullet, L. Kondic, and K. Mischaikow, Phys. Rev. E 90, 052203 (2014).
[45] M. Kramár, A. Goullet, L. Kondic, and K. Mischaikow, Phys. D 283, 37 (2014).

[46] L. A. Pugnaloni, C. M. Carlevaro, M. Kramár, K. Mischaikow, and L. Kondic, Phys. Rev. E 93, 062902 (2016).

[47] L. Kondic, M. Kramár, L. A. Pugnaloni, C. M. Carlevaro, and K. Mischaikow, Phys. Rev. E 93, 062903 (2016).

[48] V. Robins and K. Turner, Physica D 334, 99 (2016).

[49] H. Edelsbrunner and J. Harer, Computational Topology: An Introduction (American Mathematical Society, Providence, RI, 2010).

[50] C. Maria, J.-D. Boissonnat, M. Glisse, and M. Yvinec, in International Congress on Mathematical Software (Springer, Seoul, 2014), pp. 167-174.

[51] D. Morozov, DIONYSUS, a C++ library for computing persistent homology (2007).

[52] U. Bauer, M. Kerber, and J. Reininghaus, PHAT, a software library for persistent homology (2012).

[53] B. T. Fasy, J. Kim, F. Lecci, and C. Maria, arXiv:1411.1830v2 (2015).

[54] P. Dillmann, G. Maret, and P. Keim, J. Phys.: Condens. Matter 24, 464118 (2012). 